\title{
Cooling by melting precipitation in Alpine valleys: An idealized numerical modelling study
}

\author{
By S. UNTERSTRASSER and G. ZÄNGL* \\ Meteorologisches Institut der Universität München, Munich, Germany
}

(Received 22 July 2005; revised 3 April 2006)

\section{SUMMARY}

In this study, cooling by melting precipitation in Alpine valleys is investigated. This phenomenon can occur if the initial freezing level is sufficiently far below the crest height of the surrounding mountain ridges so that the air mass in the valley can become decoupled from the environmental flow. In this case, the latent heat required for melting the falling snow is continuously removed from the valley air until the snowline reaches the valley bottom. An analytical heat budget calculation indicates that the reduction of the air volume related to the mountains encompassing a valley significantly reduces the amount of precipitation needed to cool the air in a valley compared to a plain region. However, the impact of this so-called volume effect on the precipitation amounts is smaller than the geometrical volume factor frequently considered in the context of valley-wind circulations. Numerical simulations with a highly idealized two-dimensional model indicate an even smaller volume effect, which might be partly due to numerical inaccuracies. We also examined the impact of an air-mass exchange between the upper part of the valley atmosphere and the environment. It is found that the warm-air advection resulting from this air-mass exchange induces a bifurcation in the temperature evolution of the valley atmosphere. If the temperature advection exceeds a certain threshold value depending on the precipitation intensity and on the height of the initial freezing level, no significant cooling is obtained. For weaker advective warming, the valley atmosphere is cooled and the freezing level eventually reaches the valley bottom if the precipitation lasts long enough. The transition zone between the two regimes is fairly narrow, implying that forecasting real events of cooling by melting is subject to a large uncertainty. Our results also suggest that resolving the turbulent eddies that form below the melting layer is important for realizing the volume effect, which implies that mesoscale numerical models with parametrized turbulence will systematically underestimate the cooling effect in Alpine valleys.

KEYWORDS: Latent heat of fusion Snow Volume factor

\section{INTRODUCTION}

When precipitation falls into a subsaturated atmosphere, the air is cooled by evaporation until saturation is reached. The resulting temperature equals the psychrometric wet-bulb temperature if advective air-mass changes are absent. Additional cooling of the atmosphere may occur below the freezing level due to melting precipitation until an isothermal layer with near-freezing temperatures extends down to the ground and the precipitation no longer melts. A precondition for this 'cooling by melting' is that the air mass in which the falling snow or graupel melts is separated from the air mass in which large-scale lifting generates the precipitation, so that the associated latent heat is continuously removed from the same air mass. This condition is most easily fulfilled in deep mountain valleys, where the surrounding ridges tend to protect the valley atmosphere from the ambient winds. Therefore, this phenomenon is most often observed in mountainous regions (e.g. Jaffé 1966; Steinacker 1983), though it might also occur in plain regions under suitable ambient conditions. If a sufficient amount of precipitation falls, the final snowline might reach the valley bottom even though the snowline in the adjacent plains is $1000 \mathrm{~m}$ or even more above valley level. Orographic intensification of precipitation might also play an important role in this context. A notable cooling effect sometimes also occurs during heavy frontal precipitation (e.g. Wexler et al. 1954), if the frontal uplifting that generates the precipitation is mainly restricted to layers above the local freezing level. Wexler et al. (1954) present a simple heat budget calculation

* Corresponding author: Meteorologisches Institut der Universität München, Theresienstraße 37, D-80333 München, Germany. e-mail: guenther@meteo.physik.uni-muenchen.de

(c) Royal Meteorological Society, 2006. 
to estimate the amount of precipitation required to lower the melting layer by a certain amount.

It has been noted for a long time that the latent heat consumption associated with melting snow can give rise to appreciable cooling of the surrounding atmosphere (e.g. Čadež 1939). Findeisen (1940) recognized that the diabatic cooling occurring within the melting layer destabilizes the atmosphere below, giving rise to downward convection and the formation of fractocumulus clouds. Moreover, he found that intense stratiform precipitation is able to form an isothermal layer at nearly $0{ }^{\circ} \mathrm{C}$ and a depth of several hundred metres. More recently, Lin and Stewart (1986) and Szeto et al. (1988) performed numerical simulations to investigate the dynamical effects of the diabatic cooling occurring in the melting layer. Specifically, they focused on a baroclinic zone in which the freezing level intersects the ground, implying that the diabatic cooling related to melting snow is spatially inhomogeneous. They found that this setting induces a secondary circulation with rising motion near the snow-rain boundary and inferred that the precipitation intensity might be enhanced as a consequence. Szeto et al. (1988) also reported that the melting effect in combination with differential temperature advection can lead to the formation of very deep $(\gtrsim 1 \mathrm{~km})$ isothermal layers at $0^{\circ} \mathrm{C}$. In addition, Szeto and Stewart (1997) found that melting can accelerate frontogenesis, particularly when the melting layer is close to the surface.

However, only few studies exist on cooling by melting in mountain valleys although this phenomenon can have a drastic impact on local weather. Jaffé (1966) described an impressive case in which Innsbruck (western Austria, $580 \mathrm{~m}$ above sea level) received $15 \mathrm{~cm}$ of snow in June, following a day with a maximum temperature of $27^{\circ} \mathrm{C}$. On that day ( 8 June 1956), the freezing level ranged between $1200 \mathrm{~m}$ and $1500 \mathrm{~m}$ in the northern Alpine foreland. A similar case was reported by Steinacker (1980). Steinacker (1983) refined the heat budget calculation of Wexler et al. (1954) by noting that the required amount of precipitation is smaller in a valley system than over a plain due to the so-called volume effect. This effect can be quantified by the volume factor, i.e. the ratio by which the air volume in a valley is reduced compared to that over a plain due to the presence of surrounding mountain ridges. Steinacker argued that the precipitation amount required in a valley is reduced by the volume factor when turbulent mixing below the melting layer is fast enough to maintain a moist adiabatic lapse rate throughout the time. This constitutes another important reason why deep valleys are more favourable for the occurrence of cooling by melting than plain regions. The volume factor is also an important quantity in the theory of valley winds, explaining why the valley atmosphere is warmer than the atmosphere over the adjacent plains during the day and cooler at night (Steinacker 1984).

The primary goal of this study is to examine the dynamics associated with cooling by melting and the quantitative importance of the volume effect in more detail. We start with a refined analytical calculation of the precipitation amount required to lower the melting layer by a certain amount, considering an idealized valley geometry that allows for varying the volume factor between 1 and 2 . It will be demonstrated that the reduction of the required precipitation amount is in general less than the geometrical volume factor, although the difference is not too large. In a second step, we present numerical simulations with a highly idealized two-dimensional model that is able to explicitly resolve the convective motion induced below the melting level. We will also address the fact that the air mass in a valley is usually not completely decoupled from the ambient air motion. In practice, there will be a transition zone between the air above crest level, which is not severely affected by the mountains, and the air mass in the lower part of the valleys, which might be isolated for a suitable valley geometry. 
Our calculations indicate a high sensitivity of the cooling process to the precipitation intensity, the height of the initial freezing level, and the ambient wind speed. The remainder of this paper is structured as follows. Analytical heat budget calculations will be presented in section 2 . Section 3 provides a description of the numerical model, followed by a discussion of the results in section 4 . The main conclusions of our work are summarized in section 5 .

\section{ANALYTICAL CALCULATIONS}

The following analytical computation is intended to show how much precipitation is required to cool a given air mass to $0^{\circ} \mathrm{C}$. In a first step, we neglect the volume effect that might occur in valleys. We also assume that the air below the freezing level is isolated from the ambient flow that generates the precipitation and that evaporation of precipitation particles has already brought the atmosphere to saturation. Neglecting the heat capacity of rain, there is an energy balance between the heat storage in the atmosphere, the latent heat of the melting snow, and the condensation of atmospheric water vapour resulting from the diabatic cooling. It may be written as

$$
\int_{0}^{H} \rho_{\text {air }}\left[c_{p}\left\{T(z)-T_{0}\right\}+L_{\mathrm{v}}\left(q_{\mathrm{sat}}\{T(z)\}-q_{\mathrm{sat}}\left\{T_{0}\right\}\right)\right] \mathrm{d} z=P L_{\mathrm{s}} .
$$

In Eq. (1), $H$ denotes the height of the freezing level, and the temperature $T(z)$ is assumed to be higher than the freezing point $T_{0}$ between the ground and $z=$ $H$. Moreover, $\rho_{\text {air }}$ denotes the air density, $q_{\text {sat }}$ the saturation mixing ratio, $P$ the accumulated precipitation (in $\mathrm{mm}$ or $\mathrm{kg} \mathrm{m}^{-2}$ ), $c_{p}$ the specific heat capacity of air at constant pressure, and $L_{\mathrm{S}}$ and $L_{\mathrm{V}}$ denote the latent heats of fusion and condensation, respectively. To obtain an analytical solution of Eq. (1), we need to linearize the condensation term with the Clausius-Clapeyron equation

$$
\frac{\partial q_{\mathrm{sat}}}{\partial T}=\frac{q_{\mathrm{sat}} L_{\mathrm{v}}}{R_{\mathrm{v}} T^{2}},
$$

where $R_{\mathrm{v}}$ is the specific gas constant of water vapour. Linearization around the freezing point $T_{0}$ yields

$$
L_{\mathrm{v}}\left\{q_{\mathrm{sat}}(T)-q_{\mathrm{sat}}\left(T_{0}\right)\right\}=\frac{q_{\mathrm{sat}}\left(T_{0}\right) L_{\mathrm{v}}^{2}}{R_{\mathrm{v}} T_{0}^{2}}\left(T-T_{0}\right)=: c_{\mathrm{e}}\left(T-T_{0}\right) .
$$

Inserting $\rho_{\text {air }}=1.27 \mathrm{~kg} \mathrm{~m}^{-3}, c_{p}=1004 \mathrm{~J} \mathrm{~kg}^{-1} \mathrm{~K}^{-1}, L_{\mathrm{v}}=2.50 \mathrm{MJ} \mathrm{kg}^{-1}$ and $L_{\mathrm{s}}=$ $334 \mathrm{KJ} \mathrm{kg}^{-1}$, and assuming an ambient pressure of $1000 \mathrm{hPa}$, we obtain $c_{\mathrm{e}}=$ $694 \mathrm{~J} \mathrm{~kg}^{-1} \mathrm{~K}^{-1}$. This demonstrates that the condensation term is by no means small. Near the freezing point, $c_{\mathrm{e}}$ is about $70 \%$ of the heat capacity $c_{p}$, and it further increases with increasing temperature due to the exponential increase of the saturation vapour pressure. On the other hand, the heat capacity of water $\left(c_{\mathrm{w}}=4.19 \mathrm{KJ} \mathrm{kg}^{-1} \mathrm{~K}^{-1}\right)$ adds only a few per cent to the latent heat of melting, so that it can be neglected to a first approximation. If we finally assume that the initial vertical temperature gradient below the freezing level is linear, $\partial T / \partial z=\gamma(\gamma<0)$, we are able to solve Eq. (1) and obtain an equation that might be rewritten to the formula presented by Wexler et al. (1954):

$$
P_{\text {lin }}=-\frac{\rho_{\mathrm{air}} c^{*} \gamma}{L_{\mathrm{s}}} \frac{H^{2}}{2}
$$


TABLE 1. COMPARISON OF ANALYTICALLY AND NUMERICALLY COMPUTED PRECIPITATION AMOUNTS FOR AN INITIALLY SATURATED ATMOSPHERE WITH $\gamma=-6 \mathrm{~K} \mathrm{~km}^{-1}$ AND VARIOUS VALUES OF $H$

\begin{tabular}{ccc}
\hline Height, $H(\mathrm{~m})$, of initial freezing level & $P_{\text {lin }}(\mathrm{mm})$ & $P_{\text {num }}(\mathrm{mm})$ \\
\hline 500 & 4.8 & 5.1 \\
750 & 10.9 & 11.7 \\
1000 & 19.4 & 21.2 \\
1500 & 43.6 & 49.5 \\
\hline
\end{tabular}
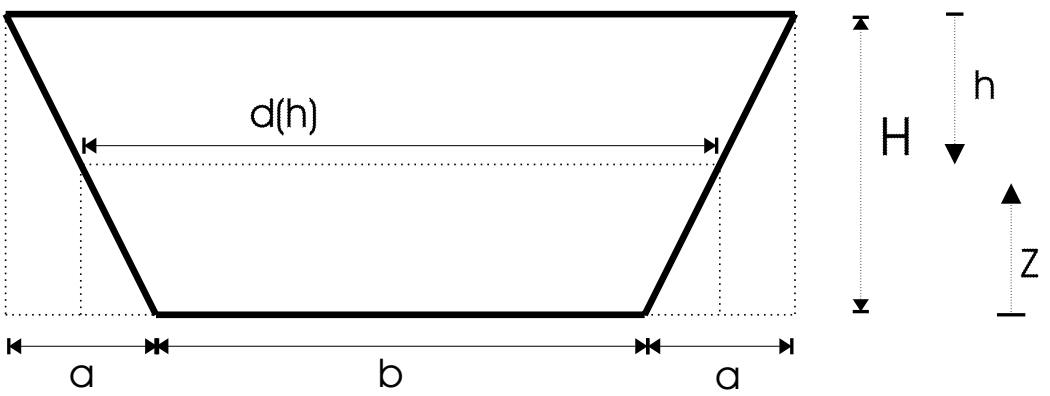

Figure 1. Idealized cross-sectional area of a valley.

Here, $c^{*}=c_{p}+c_{\mathrm{e}}$ denotes the 'effective' heat capacity of the air. Note that the required amount of precipitation increases quadratically with the height of the initial freezing level. To demonstrate the effect of linearizing the condensation term, we alternatively solved Eq. (1) numerically. The numerical result is denoted as $P_{\text {num }}$ in Table 1 . Due to the temperature dependence of $c^{*}, P_{\text {num }}$ increases faster than quadratically with $H$.

Now we generalize our computation to an idealized trapezoidal valley as depicted in Fig. 1. The height of the valley is assumed to equal the height of the initial freezing level, $H$, and the valley width at the ground is $b$. The valley widens with height to a width of $b+2 a$ at height $H$. We define $h=H-z$ and $\sigma=b / a$. Thus $\sigma=\infty$ corresponds to a plain or a valley with vertical side walls, and $\sigma=0$ indicates a triangular valley. The volume reduction factor, $f$, comparing the volume of the trapezoidal with the original rectangular case below a certain horizontal level, $\mathrm{z}$, is related to $\sigma$ as follows:

$$
f:=\frac{\text { rectangular volume }}{\text { trapezoidal volume }}=\frac{\left(b+2 a \frac{z}{H}\right) z}{\left(b+a \frac{z}{H}\right) z}=\frac{\sigma+2-2 \frac{h}{H}}{\sigma+1-\frac{h}{H}} .
$$

For the geometry under consideration, $f$ ranges between 1 and 2 , the latter corresponding to the triangular valley.

We further assume that the lapse rate, $\gamma$, corresponds to a moist neutral atmosphere and that this lapse rate is maintained below the freezing level throughout the cooling process. This implies the assumption that the convective processes excited by the cooling from above are so fast that a moist neutral atmosphere is restored instantaneously. In reality, there will be some time lag because the convective adjustment is not infinitely fast. According to this assumption, lowering the freezing level $h$ by $\mathrm{d} h$ requires that the complete air column below $h$ is cooled by $\mathrm{d} T=|\gamma| \mathrm{d} h$. The depth of the affected air column is $H-h$, so that we obtain

$$
\mathrm{d} P=\frac{1}{f} \frac{\rho_{\mathrm{air}} c^{*}|\gamma|}{L_{\mathrm{s}}}(H-h) \mathrm{d} h .
$$




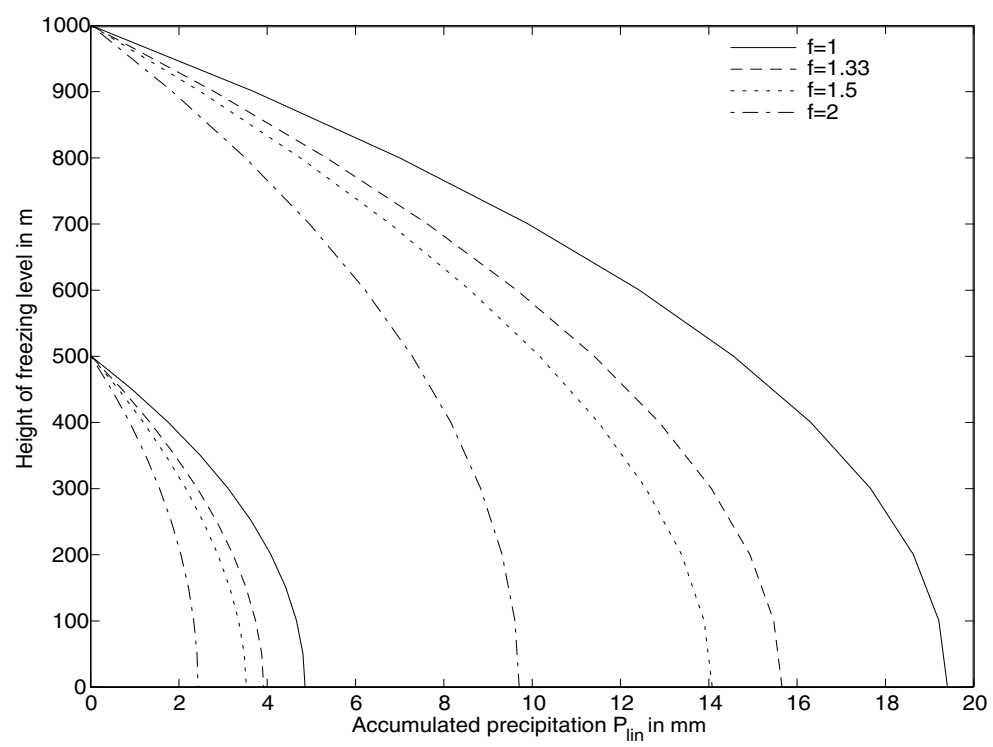

Figure 2. Height of freezing level as a function of the precipitation accumulation $P_{\text {lin }}$, for initial freezing-level heights $1 \mathrm{~km}$ and $500 \mathrm{~m}$, and volume reduction factor $f=1,1.33,1.5$ and 2 .

Introducing (5) and integrating the equation yields

$$
\frac{L_{\mathrm{s}}}{\rho_{\mathrm{air}} c^{*}|\gamma|} \int_{0}^{P} \mathrm{~d} P^{\prime}=\int_{0}^{h^{*}}(H-h) \frac{\sigma+1-\frac{h}{H}}{\sigma+2-2 \frac{h}{H}} \mathrm{~d} h,
$$

where $h^{*}$ is the current position of the freezing level. If the freezing level reaches the bottom of the valley, we have $h^{*}=H$. Introducing a non-dimensional variable $\widetilde{h}=h^{*} / H$, we finally obtain

$$
P_{\operatorname{lin}}=\frac{\rho_{\mathrm{air}} c^{*}|\gamma|}{L_{\mathrm{s}}} \frac{H^{2}}{2}\left\{\widetilde{h}-\frac{1}{2} \widetilde{h}^{2}+\frac{\sigma}{2} \widetilde{h}+\frac{\sigma^{2}}{4} \ln \left(\frac{\sigma+2-2 \widetilde{h}}{\sigma+2}\right)\right\} .
$$

The term in braces describes the reduction of $P_{\text {lin }}$ by the volume effect of the topography. Figure 2 gives an overview of the required precipitation column $P_{\text {lin }}$ for various volume factors and freezing-level heights. The graph shows $P_{\text {lin }}$ as a function of the height of the freezing level.

It is interesting to note that the reduction of the precipitation amount $P_{\text {lin }}$ is smaller than $f$ except for the limiting case of $f=2$. For example, evaluating Eq. (8) for $f=1.5$ reveals that the required precipitation amount is only reduced by a factor of 1.38 . This can be explained by the fact that the volume factor $f$ steadily decreases with progressing cooling when the width of the valley bottom $b$ (see Fig. 1) is non-zero. Since real valleys usually have a finite valley bottom, this implies that the effective volume factor relevant for cooling by melting is smaller than the volume factor relevant for valley-wind circulations (Steinacker 1984). 


\section{MODEL DESCRIPTION}

\section{(a) Model equations}

For our numerical simulations, we developed a simple two-dimensional model that allows for conducting extensive parameter studies at eddy-resolving resolution $(\lesssim 50 \mathrm{~m})$ within a reasonable amount of computing time. The model operates on a Cartesian grid in the $x-z$ plane and is derived from the incompressible Boussinesq equations by introducing a stream function, $\Psi$, via $(u, w)=\left(\Psi_{z},-\Psi_{x}\right)$. The prognostic equation for the vorticity component, $\eta$, normal to the $x-z$ plane is complemented by a minimal moisture scheme that accounts for the phase changes and related latent heats below the freezing level.

$$
\begin{aligned}
\frac{\partial \eta}{\partial t}+u \frac{\partial \eta}{\partial x}+w \frac{\partial \eta}{\partial z} & =-\frac{g}{T_{0}} \frac{\partial}{\partial x} T+\kappa \Delta \eta \\
\eta & =\nabla^{2} \Psi \\
\frac{\partial T}{\partial t}+u \frac{\partial T}{\partial x}+w\left(\frac{\partial T}{\partial z}+\frac{g}{c_{p}}\right) & =\frac{Q}{c_{p}}+\kappa \Delta T \\
\frac{\partial q_{\mathrm{s}}}{\partial t}+u \frac{\partial q_{\mathrm{s}}}{\partial x}+\left(w-v_{\mathrm{s}}\right) \frac{\partial q_{\mathrm{s}}}{\partial z} & =-M \\
\frac{\partial q_{\mathrm{r}}}{\partial t}+u \frac{\partial q_{\mathrm{r}}}{\partial x}+\left(w-v_{\mathrm{r}}\right) \frac{\partial q_{\mathrm{r}}}{\partial z} & =M+C \\
\frac{\partial q_{\mathrm{v}}}{\partial t}+u \frac{\partial q_{\mathrm{v}}}{\partial x}+w \frac{\partial q_{\mathrm{v}}}{\partial z} & =-C .
\end{aligned}
$$

In the above equations, $g$ is the gravitational acceleration, $\kappa$ is the diffusion coefficient, $q_{\mathrm{r}}, q_{\mathrm{s}}$ and $q_{\mathrm{v}}$ are the mixing ratios of rain, snow and water vapour, respectively, and $v_{\mathrm{r}}$ and $v_{\mathrm{s}}$ are the terminal fall speeds of rain and snow, respectively. The source terms in Eq. (9) describe vorticity generation due to the buoyancy related to horizontal temperature fluctuations and vorticity dissipation due to internal diffusion. The diabatic heating, $Q$, is related to the melting rate, $M$, and the condensation rate, $C$, through $Q=L_{\mathrm{v}} C-L_{\mathrm{s}} M$.

The diffusion coefficient $\kappa$ in the thermodynamic and momentum equations is implemented as

$$
\kappa_{\mathrm{h}} \frac{\partial^{2}}{\partial x^{2}}+\frac{\partial}{\partial z}\left(\kappa_{z} \frac{\partial}{\partial z}\right)
$$

with $\kappa_{\mathrm{h}}=40 \mathrm{~m}^{2} \mathrm{~s}^{-1}$ and a stability-dependent $\kappa_{z}$. The latter is set to $0.25 \mathrm{~m}^{2} \mathrm{~s}^{-1}$ if $\partial T / \partial z>-g / c_{p}$ and to $25 \mathrm{~m}^{2} \mathrm{~s}^{-1}$ if $\partial T / \partial z<-2 g / c_{p}$. In between, $\kappa_{z}$ varies linearly with $\partial T / \partial z$. The purpose of this (somewhat heuristic) implementation is to prevent the formation of strongly unstable layers near the lower model boundary without affecting the generation of resolved convection cells at higher levels. Near the lower boundary, the possible size of convective eddies is limited by the distance from the ground, so that the model is not able to resolve the eddies in this region. Therefore, vertical mixing must be parametrized there. On the other hand, applying significant vertical diffusion as soon as $\partial T / \partial z$ falls below the moist adiabatic gradient turned out to disturb the generation of resolved eddies.

The fall speeds of snow and rain are assumed to be $v_{\mathrm{s}}=1.5 \mathrm{~m} \mathrm{~s}^{-1}$ and $v_{\mathrm{r}}=$ $4.5 \mathrm{~m} \mathrm{~s}^{-1}$. Varying these values by a factor of 1.5 does not have a significant impact on the results. The condensation rate is parametrized as $C=\left(q_{\mathrm{v}}-q_{\mathrm{sat}}\right) / \tau$ with $\tau=100 \mathrm{~s}$ 
in the case of subsaturation (evaporation) and $\tau=\Delta t$ (the integration time step; see below) in the case of supersaturation. This setting is motivated by the fact that supersaturation is converted almost immediately into cloud droplets (since condensation nuclei are always abundant in reality), whereas raindrops do not evaporate instantaneously in a subsaturated atmosphere. The melting rate is parametrized as $M=0.03 q_{\mathrm{s}}\left(T-T_{0}\right)$ if the temperature is above freezing. The factor of 0.03 has been determined through experimentation such that $99 \%$ of the initial snow is melted by $T \approx 1.5^{\circ} \mathrm{C}$, guided by the observational experience that snow turns into rain near $T=1.5^{\circ} \mathrm{C}$ under saturated conditions (e.g. Steinacker 1983). Due to the cooling-induced stabilization of the melting layer, this results in a typical melting-layer depth of 300-400 m, which is consistent with radar observations (bright band) for moderate precipitation (Fabry and Zawadzki 1995).

\section{(b) Numerical implementation}

As already mentioned, the equations are discretized on a Cartesian grid in the $x-z$ plane in our model. The horizontal mesh size $\Delta x$ is $50 \mathrm{~m}$, and the vertical mesh size $\Delta z$ ranges between $20 \mathrm{~m}$ and $50 \mathrm{~m}$ depending on the steepness of the sloping side walls (see below), which are implemented as steps in the model grid. Tests with vertical side walls and various values of $\Delta z$ have shown that the results are not affected significantly by the choice of $\Delta z$. A schematic depiction of the model domain is shown in Fig. 3. The model represents a cross-section of a trapezoidal valley (areas B-D) and the lowermost part of the overlying (ambient) atmosphere (area A). The shape of the valley part varies between rectangular and triangular and attains volume factors of 1 , 1.33, 1.5 and 2 (see Eq. (5)). The total height $L_{z}$ is set to $2000 \mathrm{~m}$, and the height of the ridge level $H_{\mathrm{R}}$ ranges between $400 \mathrm{~m}$ and $1500 \mathrm{~m}$. The initial freezing level $H$ is less than or equal to $H_{\mathrm{R}}$. The valley width at ridge level $L_{x}$ is $5 \mathrm{~km}$ in the reference case with a rectangular valley but ranges between 2 and $5 \mathrm{~km}$ for trapezoidal valleys in order to achieve the desired volume factors with simple grid steps at the lateral boundaries. The number of model grid points, $(j j+1) \times(k k+1)$, is $101 \times 41$ in the reference case but differs from this value when $L_{x}$ or $\Delta z$ is changed.

Second-order centred differences are used in the model interior, except for the vertical advection of rain and snow for which we use a first-order upstream scheme so as to ensure a positive definite mass-conserving solution. At the lateral boundaries, second-order one-sided differences are used. In addition to what is stated in the above equations, a fourth-order numerical diffusion with a diffusion coefficient of $7500 \mathrm{~m}^{4} \mathrm{~s}^{-1}$ is used for Eqs. (12)-(14). Time stepping is done with a third-order Adams-Bashforth scheme for advection and diffusion terms and with a first-order Euler-forward scheme for source/sink terms. The inversion of the vorticity (Eq. 10) is accomplished with an iterative method called successive over-relaxation (SOR; Press et al. 1992), which has been modified to allow for non-rectangular grids. The time step $\Delta t$ ranges between $1 \mathrm{~s}$ and $2.5 \mathrm{~s}$ depending on $\Delta z$ such that the Courant-Friedrichs-Lewy stability threshold is reached for a horizontal or vertical wind speed of $20 \mathrm{~m} \mathrm{~s}^{-1}$. Thus, $\Delta t$ is an order of magnitude larger than in common large-eddy simulation models solving the unfiltered non-hydrostatic equations.

Initially, the model atmosphere is at rest and has a constant lapse rate $\gamma=$ $-6 \mathrm{~K} \mathrm{~km}^{-1}$, which is marginally moist stable for the temperature range considered in this study. The latter assumption is guided by the fact that warm-season coldfront passages (which initiate the most prominent cases of cooling by melting; see introduction) usually establish an approximately moist adiabatic stratification 


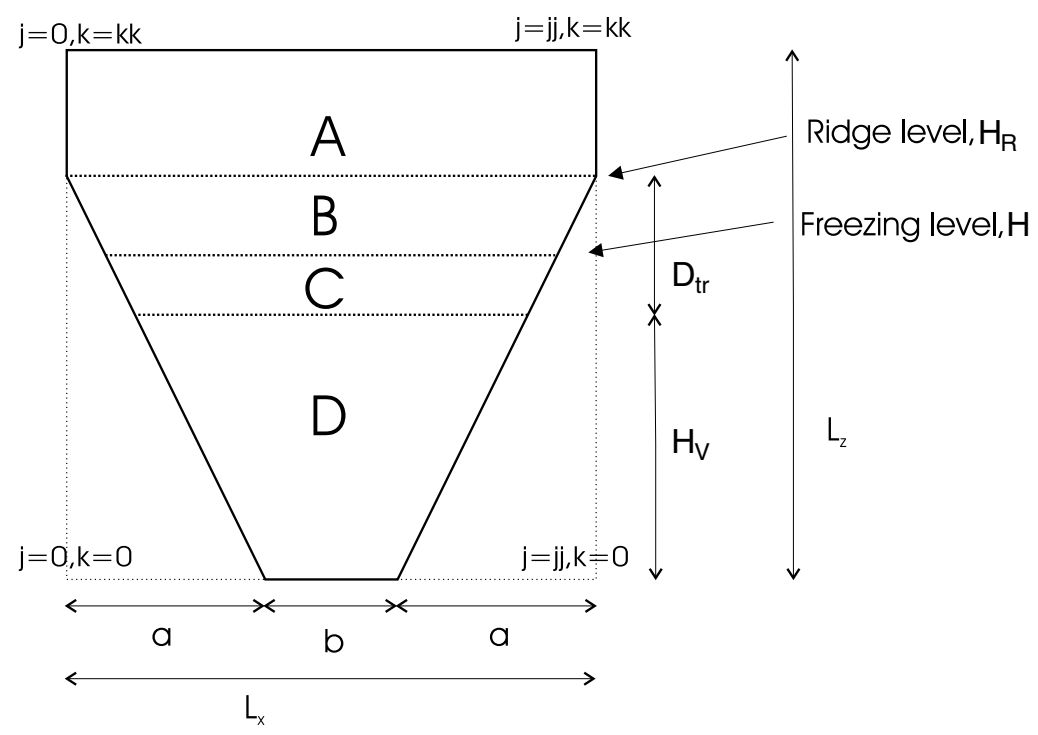

Figure 3. Schematic depiction of the model domain.

(e.g. Ralph et al. 2005). We furthermore assume that evaporative cooling has already brought the valley atmosphere to saturation. Since a few millimetres of precipitation are sufficient to almost saturate the atmosphere (except in cases of strong turbulent winds, which are not of interest here), this assumption is also meaningful and does not impose a significant restriction on the applicability of our results. To allow for a smooth model spin-up, we specify that there is initially no rain or snow inside the domain. Snow is prescribed at the top row $k=k k$ and is advected into the domain with its terminal speed $v_{\mathrm{s}}$. The snow mixing ratio is $q_{\mathrm{s}}(j, k k)=q_{\mathrm{s} \_a v g}[1+0.2\{r(j)-0.5\}]$ where $r \in[0,1]^{j j+1}$ is an array of random numbers generated at each time step. The precipitation rate $R$, which will be used in the remainder of this paper to characterize the precipitation intensity, is given by $R=\rho_{\text {air }} q_{\mathrm{s} \_ \text {avg }} v_{\mathrm{s}}$. Note that the random noise in the snowfall intensity is the only trigger for the development of convection cells below the freezing level. The fluctuations in the snowfall intensity induce spatial temperature inhomogeneities as soon as melting has started and thus create vorticity according to Eq. (9).

At all lateral model boundaries, no-slip conditions are specified, and $\Psi$ is assumed to be constant (zero) along the boundaries. This is justified at true side walls (below ridge level) and at the bottom boundary because there is surface friction in reality. For the boundaries of area A in Fig. 3 (including the top boundary), it is self-consistent with the assumptions made in our model because there is no source of air motion above the freezing level. The vorticity $\eta$ along the lateral boundaries is computed using secondorder one-sided differences under the assumption that both wind components vanish at the boundary grid points. Diffusion transmits the friction-induced vorticity into the model interior where it is able to affect $\Psi$ and thus the model dynamics.

\section{(c) Parametrized large-scale temperature advection}

So far, our model assumptions imply that there is no exchange between the valley atmosphere and the environmental air. This is not very realistic since the generation of precipitation requires the presence of ambient airflow, which in practice penetrates at least into the upper part of a valley. Unfortunately, implementing open lateral boundary 
conditions in part A of the model domain turned out to be beyond the capacity of the model numerics. Therefore, we parametrize the effect of penetrating ambient flow with a relaxation of the actual temperature towards an environmental temperature profile that equals the initial profile. We prescribe a simple Newtonian relaxation with a time constant $\tau_{\mathrm{adv}}=L_{x} / U$, where $U$ represents the (constant) ambient wind speed above $H_{\mathrm{R}}$. In an adjacent transition zone, corresponding to segments $\mathrm{B}$ and $\mathrm{C}$ in Fig. 3, $U$ is assumed to decrease linearly with height to reach zero at $z=H_{\mathrm{R}}-D_{\mathrm{tr}}=H_{\mathrm{V}}$. Below $z=H_{\mathrm{V}}$, the valley atmosphere is assumed to be isolated from the ambient flow. Regarding the realism of this highly idealized approach, we note that ambient moist neutral flow descending into a valley will maintain an approximately moist adiabatic gradient in the presence of sufficiently strong precipitation. Thus, a relaxation towards the initial temperature profile is reasonable. The assumption of a fixed penetration depth might be less realistic as strong winds tend to penetrate deeper into a valley than weak winds, but tests with a variable penetration depth did not indicate a qualitative change in the model behaviour.

The size and shape of the model domain is fixed for the experiments with parametrized warming. Specifically, $L_{x}=2 \mathrm{~km}, L_{z}=2 \mathrm{~km}, H_{\mathrm{R}}=1.5 \mathrm{~km}, \Delta x=50 \mathrm{~m}$, $\Delta z=25 \mathrm{~m}, a=750 \mathrm{~m}, b=500 \mathrm{~m}$, and the ensuing volume reduction factor $f$ is 1.6 for a reference height of $1.5 \mathrm{~km}$. Unless mentioned otherwise, the transition depth $D_{\mathrm{tr}}$ equals $500 \mathrm{~m}$, so that $H_{\mathrm{V}}=1 \mathrm{~km}$. The initial freezing level $H$ ranges between $H_{\mathrm{R}}$ and $H_{\mathrm{V}}$. Note that for $H<H_{\mathrm{V}}$, the parametrized warming would not have any impact on the cooling process. On the other hand, consideration of $H>H_{\mathrm{R}}$ is not needed because significant cooling by melting is very unlikely when the large-scale freezing level is above crest height. In this case, the ambient flow tends to participate in the large-scale lifting that generates the precipitation, so that the effect of cooling by melting does not accumulate in this air mass.

\section{Model RESUlts}

\section{(a) Cooling by melting in the absence of temperature advection}

The discussion of the model results starts with the experiments without parametrized large-scale temperature advection. This implies that the valley atmosphere below the initial freezing level is assumed to be dynamically isolated from the environment. Such conditions might be approximately fulfilled in reality if the initial freezing level is already far below the crest height of the surrounding mountain ridges. For this setting, we investigate the dependence on the volume factor of the precipitation amount required to lower the freezing level from its initial height to the valley bottom. In addition, the evolution of the cooling process is discussed.

The initial vertical temperature gradient is assumed to be $\gamma=-6 \mathrm{~K} \mathrm{~km}^{-1}$, and the relative humidity is $100 \%$. The average precipitation intensity entering the model domain from the top is $R=5 \mathrm{~mm} \mathrm{~h}^{-1}$. The simulations are carried out until the $0{ }^{\circ} \mathrm{C}$ line reaches the bottom. We consider initial freezing levels $H$ of $400 \mathrm{~m}, 600 \mathrm{~m}, 800 \mathrm{~m}$, $1 \mathrm{~km}$ and $1.5 \mathrm{~km}$ and volume factors $f$ of $1,1.33,1.5$ and $2 . H$ equals $H_{\mathrm{R}}$ in all cases discussed here. The results are displayed in Fig. 4.

The shape of the cooling curves obtained from the numerical model is broadly similar to the analytic ones (Fig. 2), but a systematic discrepancy appears in the initial phase of the cooling process. While the analytic calculations indicate a smooth and continuous cooling process, the model produces a rapid cooling at the beginning followed by a phase in which the freezing level almost stagnates. This can be explained by the fact that the numerical model needs a finite amount of destabilization to initiate 

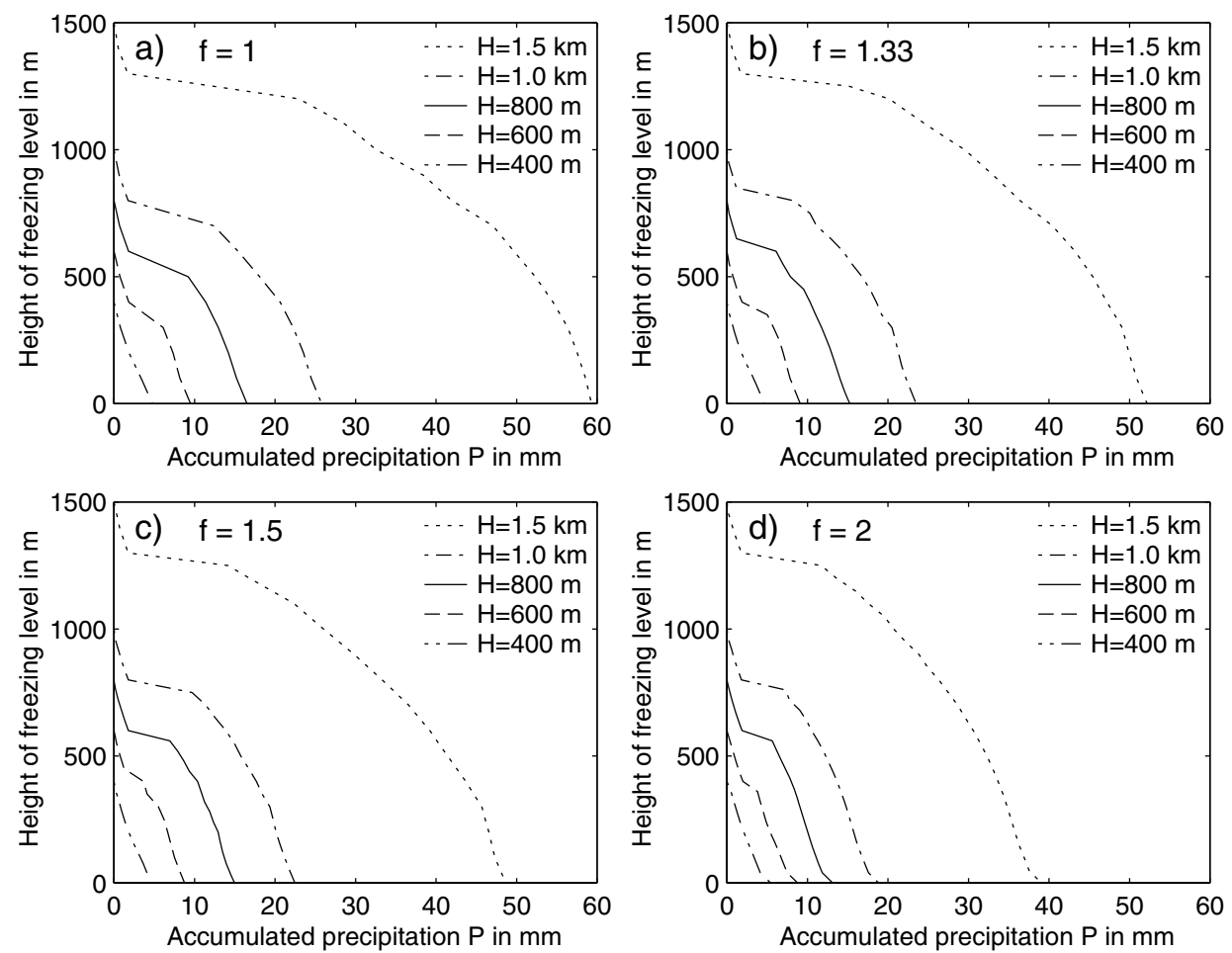

Figure 4. Necessary precipitation amounts as a function of the height of the freezing level. The rain rate $R$ is set to $5 \mathrm{~mm} \mathrm{~h}^{-1}$, implying that the range of the $x$-axis corresponds to 12 hours. The parameters are the initial height of the freezing level $H(400 \mathrm{~m}, 600 \mathrm{~m}, 800 \mathrm{~m}, 1 \mathrm{~km}$ and $1.5 \mathrm{~km})$ and the volume reduction factor $f(1,1.33,1.5$ and 2). Precipitation is evaluated at the valley bottom.

convective cells below the freezing level. Recall that the model atmosphere is initially at rest and that air motions are generated only due to spatial inhomogeneities in the melting rate. Once the convection cells are established, the initial cooling is distributed through the volume below the freezing level until a nearly moist neutral stratification is restored. Afterwards, the simulated cooling progresses in a similar way to the analytic model.

A quantitative comparison of the final precipitation amounts with the analytical ones (Fig. 2) reveals that the numerical model predicts systematically higher amounts than the analytical theory. Part of this discrepancy is due to the linearization of the condensation term (Eq. (3)), but comparing the results for $f=1$ (Fig. 4(a)) with the $P_{\text {num }}$ values provided in Table 1 indicates that there are additional factors. One reason is that the precipitation amounts displayed in Fig. 4 are measured at the ground and thus contain a contribution of the condensation term. Since the model does not include cloud water, the water vapour removed from the atmosphere during the cooling process is immediately converted into rain, which adds about $3 \mathrm{~mm}$ of precipitation in the case of $H=1.5 \mathrm{~km}$. Another contribution arises from the finite depth of the melting layer $(\sim 300 \mathrm{~m})$ in the numerical model. The final phase of the cooling process takes longer in the model than in the analytical computation because part of the precipitation reaches the ground as snow and thus does not contribute to atmospheric cooling. In addition, the numerical diffusion and discretization errors might slow down the cooling process in the numerical model. 


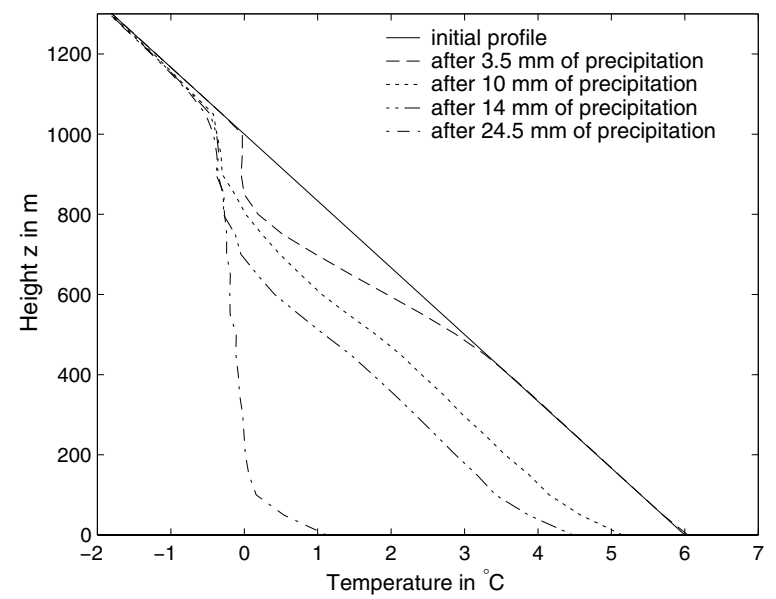

Figure 5. Evolution of the temperature profile in the experiment with $H=1 \mathrm{~km}, L_{x}=5 \mathrm{~km}, L_{z}=2 \mathrm{~km}$ and $f=1$, after $0,3.5,10,14$ and $24.5 \mathrm{~mm}$ of precipitation.

Another important difference between the numerical and analytical models is that the impact of the volume factor is systematically smaller in the numerical model. For example, the analytical precipitation ratio for $f=1.5$ is 1.38 while the numerical one is only about 1.24. An even larger difference is found for $f=2$, for which the analytical precipitation ratio is 2 whereas the numerical one is only 1.56. A contribution to this discrepancy arises from the condensation of atmospheric water vapour, which does not depend on the volume factor. Moreover, the effect of the finite melting-layer depth does not decrease with the volume factor. The particularly large discrepancy in the case of $f=2$ may be explained by numerical inaccuracies occurring in the lower part of the valley. Due to its triangular shape, this part of the valley is represented by very few grid points, so that the model is unable to maintain convective cells as the freezing level approaches the valley bottom. Thus, the vertical mixing is solely accomplished by the explicit diffusion term, which apparently does not enable the model to properly represent the volume effect. In fact, the simulated volume effect is negligible for $H=400 \mathrm{~m}$. On the other hand, the quasi-horizontal segment of the cooling curves starting at $H=1500 \mathrm{~m}$ is only about half as long for $f=2$ as for $f=1$, implying that the model reproduces the volume effect if enough grid points are available to generate convection cells.

The evolution of the temperature profile during the cooling process is illustrated in Fig. 5 for the case $H=1 \mathrm{~km}, f=1$. The profile after $3.5 \mathrm{~mm}$ of precipitation represents the quasi-horizontal segment of the cooling curves displayed in Fig. 4. An isothermal layer has already formed at the freezing level, and growing convective motions are transmitting the cooling signal to lower levels. Later on, an approximately moist neutral profile is restored, and the isothermal layer continues to grow until it reaches the ground. Overshooting convective motion and the explicit temperature diffusion keep the temperature of the isothermal layer slightly below freezing.

Figure 6(a) shows a snapshot of the circulation pattern during the cooling process. Data are taken from the same case as in Fig. 5 after $10 \mathrm{~mm}$ of precipitation, corresponding to a freezing level of about $800 \mathrm{~m}$. It is evident that the convective motion is largely restricted to the moist neutral layer below the freezing level, but the most intense cells slightly penetrate into the isothermal layer. Individual convection cells are unsteady 

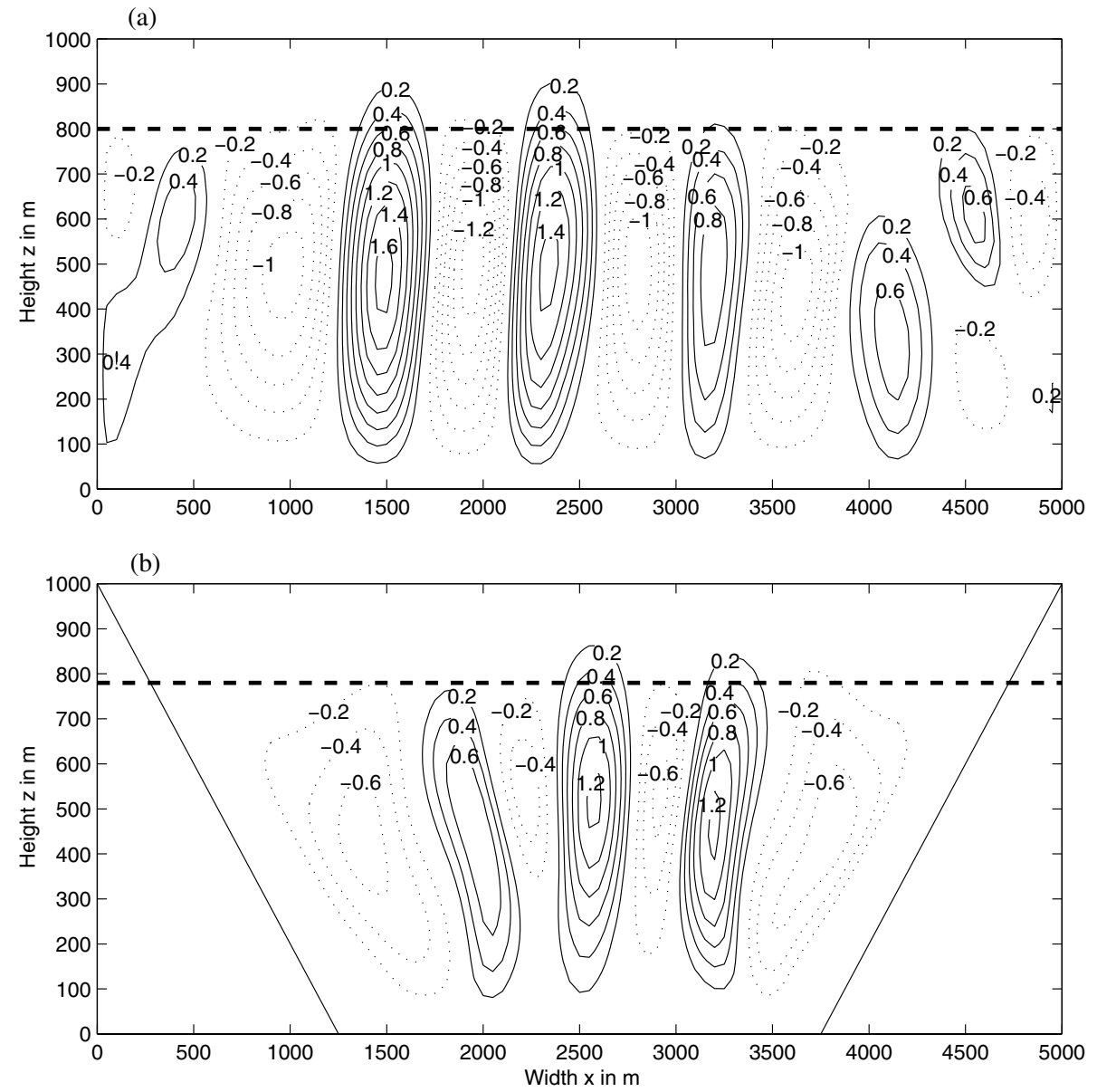

Figure 6. Vertical wind field after $10 \mathrm{~mm}$ of precipitation for experiments with $H=1 \mathrm{~km}, L_{x}=5 \mathrm{~km}$, $L_{z}=2 \mathrm{~km}$ and (a) $f=1$, (b) $f=1.33$. Solid (dashed) contours denote positive (negative) vertical winds with an interval of $0.2 \mathrm{~m} \mathrm{~s}^{-1}$. The bold dashed line indicates the freezing level.

because of their inherent instability and the random forcing (see section 3(b)), but statistical measures like their average spacing and average intensity remain quasi-steady between about $5 \mathrm{~mm}$ and $13 \mathrm{~mm}$ of precipitation. Afterwards, the intensity gradually decreases as the freezing level approaches the ground. At the time shown here, the maximum vertical wind speed is $1.6 \mathrm{~m} \mathrm{~s}^{-1}$ for upward motion and $1.2 \mathrm{~m} \mathrm{~s}^{-1}$ for downward motion. However, fluctuations of the maximum speed are substantial, ranging between 1.1 and $1.8 \mathrm{~m} \mathrm{~s}^{-1}$ for upward motion and between 0.7 and $1.3 \mathrm{~m} \mathrm{~s}^{-1}$ for downward motion during the quasi-steady phase (not shown). The width of the convection cells ranges between 400 and $700 \mathrm{~m}$, which indicates that it is not determined by the mesh size of the model $(50 \mathrm{~m})$. Sensitivity experiments with halved horizontal diffusion (i.e. $\left.\kappa_{\mathrm{h}}=20 \mathrm{~m}^{2} \mathrm{~s}^{-1}\right)$ and/or halved mesh size $(25 \mathrm{~m})$ did not indicate a significant change in the cell structure. We also tested the impact of several other model parameters on the cooling behaviour and the statistical properties of the convection cells. Varying the fall speeds of snow and rain and the melting rate (melting-layer depth) within the observed range of variability does not have a significant impact. However, decreasing the time constant $\tau$ for evaporating rain in a subsaturated atmosphere intensifies and narrows 
the updraughts because the temperature difference between the up- and downdraughts increases, which in turn leads to stronger overshooting and a colder quasi-isothermal layer. For $\tau=10 \mathrm{~s}$, this layer is established at about $-1{ }^{\circ} \mathrm{C}$ rather than near the freezing point (Fig. 5), which is less consistent with observations (e.g. Findeisen 1940).

The corresponding cell structure in a valley with $f=1.33$ is displayed in Fig. 6(b). Due to the volume effect, the freezing level is about $20 \mathrm{~m}$ lower than for $f=1$. The maximum wind speeds at the selected point of time (10 $\mathrm{mm}$ of precipitation) are weaker than for $f=1$, but the average spacing of the convection cells is similar. The temporal evolution of the wind field reveals larger fluctuations than for $f=1$, which is presumably because the narrowing valley supports a smaller number of individual cells. However, the average intensity of the circulation is of similar magnitude.

\section{(b) Experiments with parametrized temperature advection}

In reality, the ambient flow penetrates at least partly into a valley. If the large-scale freezing level is below crest level, which is a precondition for the occurrence of significant cooling by melting, the ambient flow is hardly affected by the diabatic cooling related to melting snow. Penetration of the ambient air mass into a valley thus constitutes a positive temperature advection unless there is large-scale cold-air advection. In the frame of our idealized model, this temperature advection is parametrized with a Newtonian relaxation of the valley temperature towards the initial profile. A warming with similar properties may also occur when a down-valley drainage flow develops within the cooled air mass, leading to compensating subsidence of ambient air (e.g. Steiner et al. 2003). Unless mentioned otherwise, we assume that the advecting wind speed $U$ decreases linearly between $z=1500 \mathrm{~m}$ (ridge level) and $z=1000 \mathrm{~m}$, and that the initial freezing level $H$ lies between these heights. The shape of the valley and thus the volume factor remains fixed in the following experiments, but $R, U$ and $H$ are varied.

Due to the geometry of the problem, varying $H$ or $U$ can be expected to introduce a bifurcation between the occurrence and non-occurrence of significant cooling by melting. If $U$ is too strong and/or $H$ is too high, the latent heat of melting is offset by the relaxation term. In this case, the underlying air mass will not experience any significant cooling. If, however, the freezing level manages to penetrate far enough down into the valley, then cooling by melting will continue and the freezing level will eventually reach the valley bottom. We therefore define a critical height $H_{\mathrm{c}}$ denoting the maximum value of $H$ that, for a given rain rate $R$, leads to significant cooling inside the valley. Our criterion for significant cooling is that the freezing level falls below $z=1000 \mathrm{~m}$ or, alternatively, the surface temperature decreases by at least $1.5 \mathrm{~K}$ within the simulation time. The second condition is relevant if $H$ is above $1250 \mathrm{~m}$. Since we assume a constant lapse rate $\gamma$ of $-6 \mathrm{~K} \mathrm{~km}^{-1}$, a temperature drop of $1.5 \mathrm{~K}$ corresponds to a downward shift of the freezing level by $250 \mathrm{~m}$.

In the following series of simulations, the integration time is either set to a constant value of 10 hours or specified such that a total precipitation amount of $60 \mathrm{~mm}$ enters the model domain from the top. The precipitation rate $R$ is varied between 2 and $6 \mathrm{~mm} \mathrm{~h}^{-1}$, so that the total precipitation ranges between 20 and $60 \mathrm{~mm}$ in the first case, whereas the integration time ranges between 10 and 30 hours in the second case. Recall that $60 \mathrm{~mm}$ of precipitation are sufficient to lower the freezing level from $z=1500 \mathrm{~m}$ to the valley bottom in the absence of advective warming (Fig. 4). The advective relaxation time-scale $\tau_{\text {adv }}$ ranges between 200 and $2000 \mathrm{~s}$, corresponding to ambient wind speeds between 1 and $10 \mathrm{~m} \mathrm{~s}^{-1}$. These values sound quite low but we have to mention that the 

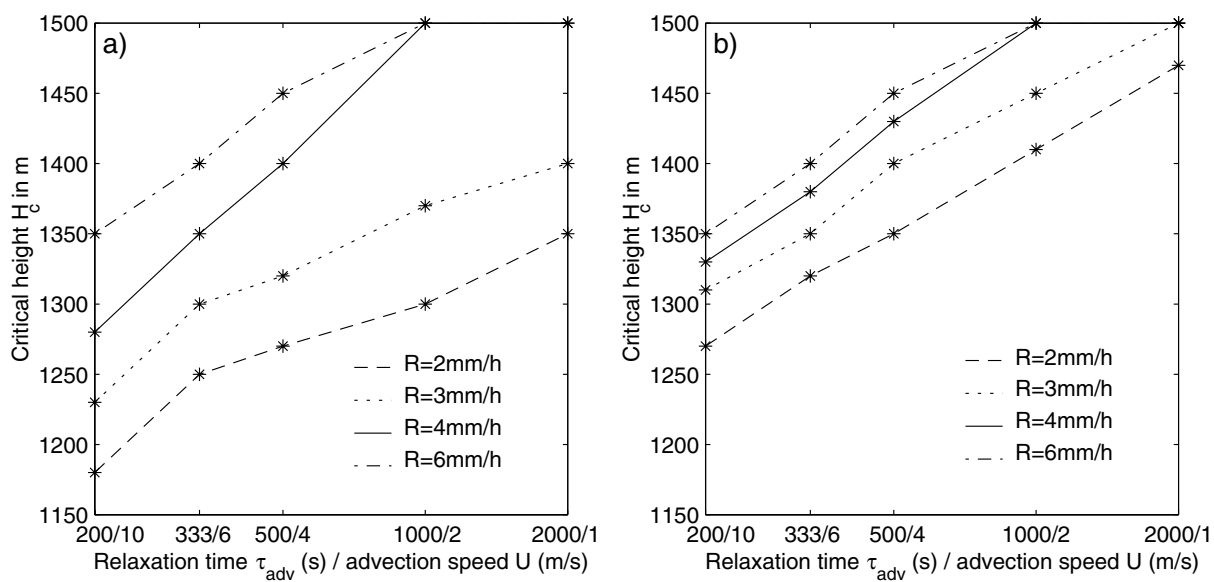

Figure 7. Relationship between critical height $H_{\mathrm{c}}$ and relaxation time $\tau_{\text {adv }}$ for various precipitation intensities $R$ for (a) fixed integration time $(10 \mathrm{~h})$, and (b) fixed total precipitation $(60 \mathrm{~mm})$.

present valley width $(2 \mathrm{~km})$ is quite small. For a valley width $L_{x}$ of $5 \mathrm{~km}$, the relaxation times would correspond to wind speeds from 2.5 to $25 \mathrm{~m} \mathrm{~s}^{-1}$.

The results of the test series are displayed in Fig. 7(a) for fixed integration time (10 hours) and in Fig. 7(b) for fixed total precipitation $(60 \mathrm{~mm})$. Note that the simulations for $R=6 \mathrm{~mm} \mathrm{~h}^{-1}$ are identical. The general feature seen in both cases is that $H_{\mathrm{c}}$ increases with increasing relaxation time (decreasing wind speed) and with increasing precipitation intensity. Both dependencies are in accordance with intuitive expectation. If the advective warming decreases for a given rate of diabatic cooling, then the critical height $H_{\mathrm{c}}$ increases. Likewise, $H_{\mathrm{c}}$ increases when the precipitation rate increases for a fixed wind speed because a stronger diabatic cooling can more easily overcome the advective warming. For $R \geqslant 4 \mathrm{~mm} \mathrm{~h}^{-1}$ and $\tau_{\text {adv }}>1000 \mathrm{~s}, H_{\mathrm{c}}$ coincides with the ridge level $(1500 \mathrm{~m})$, implying that the advection effect becomes unimportant when heavy precipitation is combined with weak advection. On the other hand, $H$ must be significantly below crest level when the advective warming (the ambient wind) is strong.

In the case of light precipitation $\left(R \leqslant 3 \mathrm{~mm} \mathrm{~h}^{-1}\right)$, comparing Figs. 7(a) and 7(b) shows that the integration time makes a significant difference. This is not surprising as a total precipitation amount of $20 \mathrm{~mm}$, as obtained for $R=2 \mathrm{~mm} \mathrm{~h}^{-1}$ and an integration period of 10 hours, is just sufficient to fulfil the threshold criterion in the absence of temperature advection for $H=1500 \mathrm{~m}$ (see Fig. 4(c), showing a drop of the freezing level by about $300 \mathrm{~m}$ for $P=20 \mathrm{~mm}$ ). To determine the impact of the precipitation intensity, it is thus necessary to keep the total precipitation amount rather than the integration time fixed. On the other hand, precipitation events usually do not last for 30 hours, so that the dependence shown in Fig. 7(a) might be more realistic. In practice, significant cooling by melting is very unlikely for light precipitation because advective effects are never exactly zero and the duration of precipitation events is limited.

To obtain more insight into the interplay between cooling by melting and advective warming, the subsequent figures present the temporal evolution of the cooling process for different parameter combinations. Figure 8 displays the evolution of the freezing level and the surface temperature at the valley bottom for different advective wind speeds. The freezing level is defined as $T=0.1{ }^{\circ} \mathrm{C}$ in Fig. 8(a) because the advective warming tends to keep the temperature of the melting layer slightly above $0{ }^{\circ} \mathrm{C}$. 

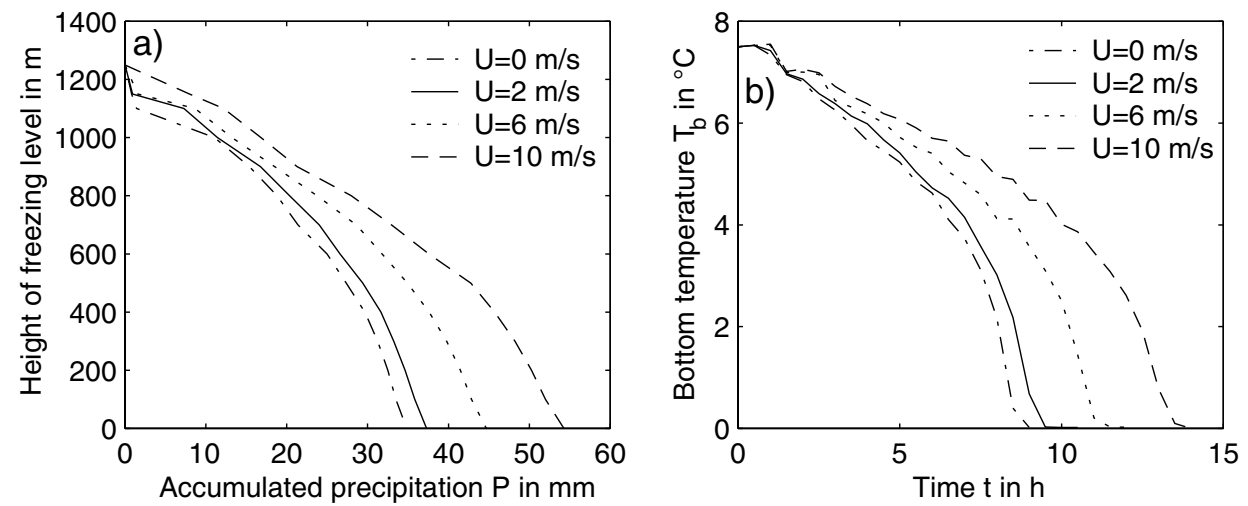

Figure 8. Temporal evolution of the cooling process for experiments with $H=1250 \mathrm{~m}, R=4 \mathrm{~mm} \mathrm{~h}^{-1}$ and advective wind speeds ranging between 0 and $10 \mathrm{~m} \mathrm{~s}^{-1}$. In (a), the freezing level is defined as $T=0.1^{\circ} \mathrm{C}$ (see text for explanation), and the accumulated precipitation shown on the abscissa is evaluated at the upper model boundary. Thus, the abscissae in (a) and (b) are equivalent.

The height of the initial freezing level $H$ and the rain rate $R$ are fixed at $1250 \mathrm{~m}$ and $4 \mathrm{~mm} \mathrm{~h}^{-1}$, respectively. According to Fig. 7, this parameter setting leads to significant cooling for all advection speeds considered here. Figure 8(a) shows that, without advection, the freezing level reaches the ground after about $35 \mathrm{~mm}$ of precipitation whereas almost $55 \mathrm{~mm}$ are needed for an advection speed of $10 \mathrm{~m} \mathrm{~s}^{-1}$. This is because the parametrized advective warming acts as an additional heat source below $H$ that melts part of the snow falling in from above. This process continues after the freezing level has reached the lower boundary of the parametrized heating $(z=1000 \mathrm{~m})$, so that the cooling curves progressively diverge with time. Regarding the realism of this implementation, it might be objected that the formation of a stable isothermal layer tends to reduce the penetration of the ambient airflow into a valley, so that the parametrized heating should decrease with progressing cooling. However, given the strong idealizations adopted in our model, it was felt that implementing such a feedback would be quite speculative and thus would not lead to additional understanding of the process. It is also worth mentioning that the snow is known to be very wet in cases of cooling by melting, leading to serious forest damage if a significant amount of snow reaches low altitudes (e.g. Jaffé 1966). This is represented as a mixture of snow and rain in our idealized model.

The corresponding temporal evolution of the bottom temperature is shown in Fig. 8(b). It is evident that the shape of the various curves is quite similar to those shown in Fig. 8(a) except for the initial spin-up phase of the convection cells. This confirms that the simulated lapse rate is close to moist neutral throughout most of the integration and suggests that the height of the freezing level and the bottom temperature can be used interchangeably to characterize the cooling process. However, in cases of very slow cooling $\left(H\right.$ close to $H_{\mathrm{c}}$ ), the temperature of the quasi-isothermal layer can be even higher than $0.1{ }^{\circ} \mathrm{C}$ (not shown). To avoid further complications in the definition of the freezing level, we thus use the bottom temperature in the subsequent figures.

In the next series of experiments, we keep $U$ constant at $6 \mathrm{~m} \mathrm{~s}^{-1}$ but vary $R$ between 2 and $10 \mathrm{~mm} \mathrm{~h}^{-1}$. Results are shown in Fig. 9(a) for $H=1250 \mathrm{~m}$ and in Fig. 9(b) for $H=1300 \mathrm{~m}$. In addition, Fig. 9(b) presents the evolution obtained for $H=1350 \mathrm{~m}$ and $R=2 \mathrm{~mm} \mathrm{~h}^{-1}$. This choice of parameters clarifies the nature of the bifurcation between the occurrence and non-occurrence of cooling by melting. It is readily evident from 

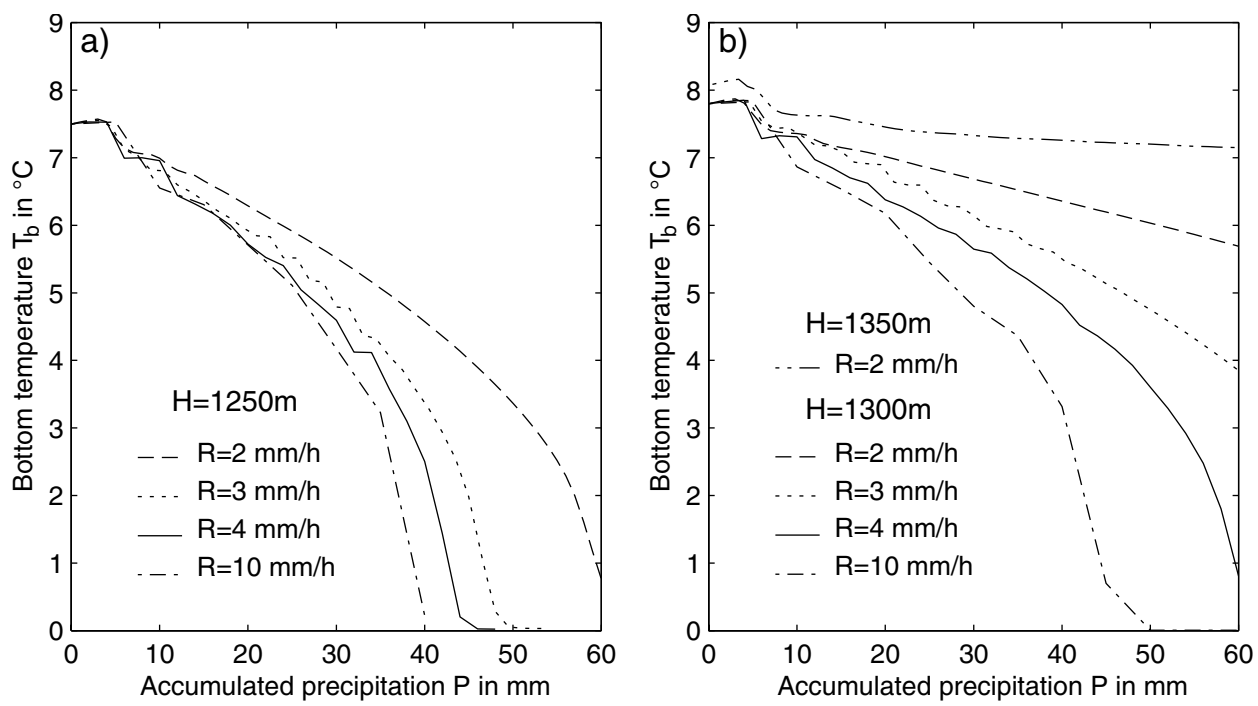

Figure 9. Temporal evolution of the cooling process for experiments with $U=6 \mathrm{~m} \mathrm{~s}^{-1}$ and (a) $H=1250 \mathrm{~m}$, (b) $H=1300 \mathrm{~m}$. The precipitation rate $R$ ranges between 2 and $10 \mathrm{~mm} \mathrm{~h}^{-1}$, and the accumulated precipitation shown on the abscissa is evaluated at the upper model boundary.

Fig. 9 that the evolution of the temperature field in the valley depends very sensitively on $H$ and $R$. For $H=1250 \mathrm{~m}$, the freezing level reaches the valley bottom in all cases, and the required precipitation amount just differs by a factor of 1.6 between rain rates of 10 and $2 \mathrm{~mm} \mathrm{~h}^{-1}$, respectively (Fig. 9(a)). However, increasing $H$ by only $50 \mathrm{~m}$ drastically enhances the sensitivity to the precipitation intensity. While there is still a reasonably rapid cooling for $R=10$ and $4 \mathrm{~mm} \mathrm{~h}^{-1}$, the cooling process becomes very slow for $R=3$ and $2 \mathrm{~mm} \mathrm{~h}^{-1}$ (Fig. 9(b)). For $H=1350 \mathrm{~m}$ and $R=2 \mathrm{~mm} \mathrm{~h}^{-1}$, the cooling process even stagnates after a slight initial cooling phase. In the latter case, $H$ is about $30 \mathrm{~m}$ above $H_{\mathrm{c}}$ (see Fig. 7(b)). A closer inspection of the corresponding model output fields reveals that the snow melting below the freezing level forms a layer of enhanced static stability (but not isothermal due to the parametrized warming) and temporarily triggers downward convection due to destabilization below, so that the underlying atmosphere experiences some cooling. However, virtually all the snow melts within the layer of parametrized warming, leading to a near-equilibrium state without sustained cooling.

To demonstrate that a bifurcation can also be obtained for moderate precipitation, Fig. 10(a) displays another series of experiments in which $U$ and $R$ are fixed at $6 \mathrm{~m} \mathrm{~s}^{-1}$ and $4 \mathrm{~mm} \mathrm{~h}^{-1}$, respectively. While the freezing level reaches the valley bottom reasonably rapidly for $H \leqslant 1300 \mathrm{~m}$, the cooling process becomes very slow for $H=1350 \mathrm{~m}$. A further increase of $H$ to $1400 \mathrm{~m}$ again yields a solution with a slight initial cooling and a subsequent stagnation of the melting layer within the layer of advective warming. This confirms that a shift of the initial freezing level by about $100 \mathrm{~m}$, corresponding to a shift of the large-scale temperature profile by $0.6 \mathrm{~K}$, can be sufficient to trigger a bifurcation between rapid cooling by melting and suppressed cooling. Alternatively, variations in the precipitation intensity by a factor of about 2 can trigger this bifurcation when $H$ is kept fixed. Finally, Fig. 10(b) shows a similar series of experiments in which the penetration depth of the ambient flow $D_{\text {tr }}$ is increased from 

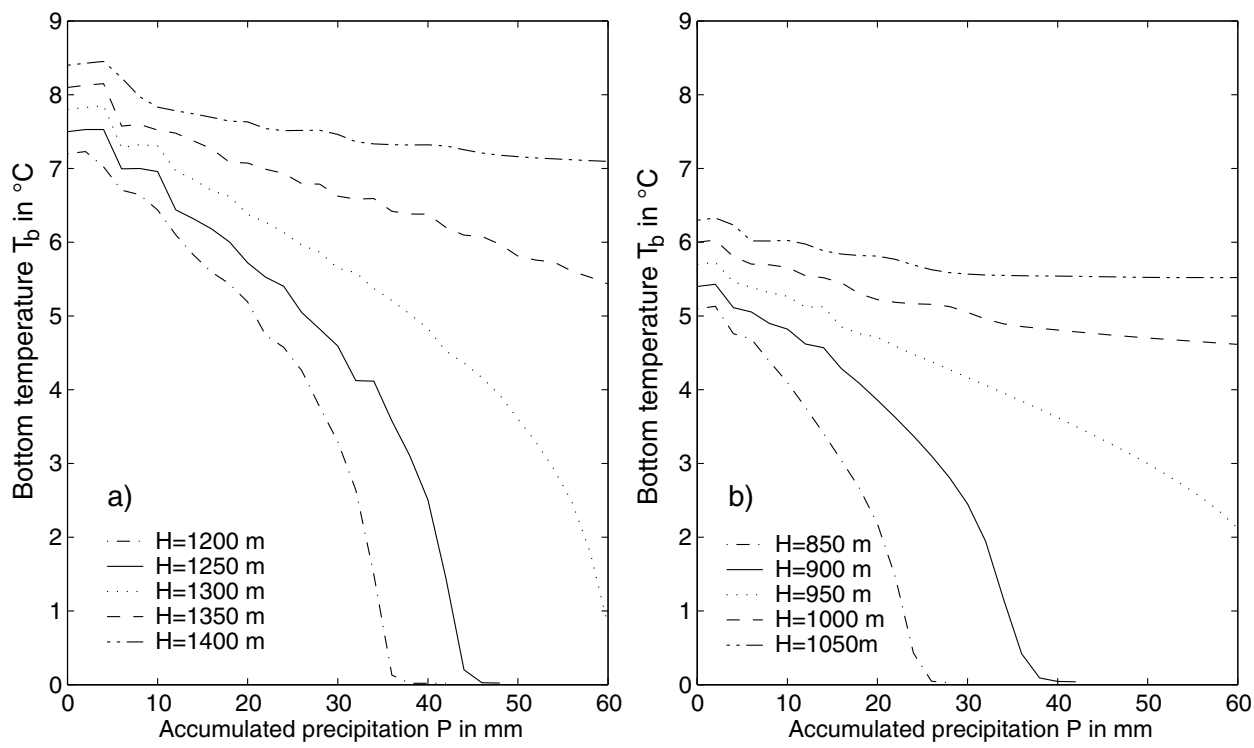

Figure 10. Temporal evolution of the cooling process for experiments with $R=4 \mathrm{~mm} \mathrm{~h}^{-1}, U=6 \mathrm{~m} \mathrm{~s}^{-1}$ and (a) $D_{\mathrm{tr}}=500 \mathrm{~m}$, and (b) $D_{\mathrm{tr}}=1000 \mathrm{~m}$. The height of the initial freezing level $H$ ranges in (a) between 1200 and $1400 \mathrm{~m}$, and in (b) between 850 and $1050 \mathrm{~m}$, and precipitation is evaluated at the upper model boundary.

$500 \mathrm{~m}$ to $1000 \mathrm{~m}$, so that the external warming reaches down to $z=500 \mathrm{~m}$. Due to the deeper flow penetration, the critical heights are generally lower than before, but the bifurcation property remains essentially unchanged. Again, a shift of the initial freezing level by $100 \mathrm{~m}$ suffices to trigger a bifurcation.

\section{(c) Implications for mesoscale modelling and weather forecasting}

An important practical consequence of these results is that forecasting cooling by melting precipitation is extremely difficult. As just discussed, an uncertainty of $100 \mathrm{~m}$ in the height of the initial freezing level can already be sufficient to obtain a completely wrong temperature evolution when the environmental parameters lie close to the critical values. In reality, the bifurcation might be even sharper because of positive feedback effects that are not accounted for in our model. For example, the penetration depth of the ambient flow can be expected to increase with its speed and to decrease with progressing cooling by melting because the stable isothermal layer forming below the initial freezing level reduces vertical mixing. Further complications of the forecasting issue arise from the large sensitivity of the cooling process to the precipitation rate and to the wind speed around the freezing level. These parameters are also subject to significant forecast uncertainties, implying that even a perfect prediction of the large-scale freezing level does not guarantee a qualitatively correct evolution of the cooling process.

Regarding the suitability of high-resolution mesoscale models, which presumably will become available for operational forecasting in the near future, further caveats arise. Even at a horizontal mesh size of order $1 \mathrm{~km}$, a model topography is significantly smoothed compared to real Alpine topography. As a consequence, the average crest level of the mountain ridges encompassing a valley might be too low, so that the penetration depth of the ambient flow is biased even in the absence of modelling uncertainties like the representation of friction-induced flow separation in the lee of a mountain ridge. 
This implies that the value of $H$ at which a mesoscale model starts to predict significant cooling by melting could be systematically too low. In addition, our idealized simulations indicate that a model needs to resolve the convective cells triggered below the melting level in order to capture the volume effect in a valley. If the vertical mixing is solely accomplished by parametrized diffusion, the volume effect cannot be properly represented because the diffusion acts primarily in the vertical direction. This suggests that large-eddy simulations are needed to quantitatively reproduce the relationship between accumulated precipitation and related atmospheric cooling whereas a mesoscale model will systematically overpredict the required precipitation amounts. Another important problem of current mesoscale models is due to the explicit numerical diffusion, which is usually computed along the terrain-following coordinate surfaces. In steep valleys, this might introduce spurious temperature tendencies that are as large as, or even larger than, the temperature tendencies due to the melting of snow. In fact, attempts to simulate observed cases of cooling by melting with the mesoscale model MM5 were unsuccessful despite using a modified numerical diffusion scheme that strongly reduces the related numerical errors (Zängl 2002). Though the precipitation amounts were quite realistic in these simulations, the melting-induced diabatic cooling did not accumulate in the valleys, probably because the exchange between the valley atmosphere and the environmental air was too strong.

We thus hypothesize that the most promising way of predicting cooling by melting in Alpine valleys is to combine local experience with model forecasts of precipitation and the large-scale freezing level. Though prominent events of cooling by melting are rare, experience shows that only a few inner-Alpine valley systems are favourable for pronounced cooling, probably because the others do not become decoupled from the ambient flow. One therefore needs to analyze previous cases of cooling by melting as well as heavy precipitation cases without pronounced cooling in order to estimate the critical environmental parameters (freezing level, precipitation intensity, etc.), which can afterwards be used for forecasting purposes. A closely related problem is predicting the snowline in cases of wintertime warm-front passages with pre-existing cold air. Such situations can also lead to large local variations in the height of the snowline, depending on whether the cold air is trapped in a specific valley segment or not. Research on this topic is in a very early stage, but a first modelling study by Zängl (2005) indicates that the predictability might be better in cases of pre-existing cold air than in cases of pure cooling by melting.

\section{Conclusions}

We presented analytical calculations and idealized numerical simulations of cooling by melting precipitation in Alpine valleys. If the initial freezing level is sufficiently far below the crest level of the surrounding mountain ranges, the air mass in a valley might become decoupled from the environmental flow generating the precipitation. The cooling related to the melting snow then accumulates in the valley air mass, so that the melting level will eventually reach the valley bottom if enough precipitation falls. The first goal of this study was to test the hypothesis of Steinacker (1983) that the precipitation amount needed to cool the atmosphere in a valley is reduced compared to that needed over a plain by the so-called volume factor. In addition, we addressed the influence of an air-mass exchange between the valley and the environment that occurs in reality at least in the upper part of a valley.

The volume factor describes the ratio by which the air volume in a valley is reduced compared to that over a plain due to the presence of the surrounding mountain ridges. 
In the context of thermally driven valley-wind circulations, this factor provides an estimate of the ratio by which the vertically integrated diurnal temperature amplitude is larger in a valley than over a plain (Steinacker 1984). Steinacker $(1983,1984)$ also pointed out that for complex valley systems with tributaries, the whole catchment area needs to be considered to get a realistic estimate of the volume factor. This usually leads to substantially larger volume factors than when considering the main valley only, often exceeding a value of 2 . Our analytical and numerical results confirm that the volume effect accelerates the cooling process in a valley compared to a plain. However, the fractional decrease of the precipitation amount required to cool the atmosphere is smaller than the vertically integrated volume factor used by Steinacker. This is partly because only the volume reduction below the local freezing level is relevant for cooling by melting, implying that the effective volume factor decreases with progressive cooling if a valley has a bottom with a finite width. In particular, volume factors near or above 2 are unlikely to occur for cooling by melting because such high values are obtained only when integrating up to crest level (Steinacker 1984). Yet, if the initial freezing level is close to the crest level, advective effects are strong and tend to suppress any significant cooling in the underlying valley. In practice, it might be difficult to determine if the protection against lateral air exchange or the volume reduction is the dominant valley effect. From a modelling perspective, it is important to note that the turbulent eddies triggered below the melting layer due to destabilization need to be explicitly resolved in a model to capture the volume effect. Parametrized turbulence systematically underestimates this effect.

Our numerical experiments with parametrized temperature advection indicate a subtle balance between cooling by melting and advective warming, which implies that slight changes in the initial or environmental conditions can trigger a bifurcation between the occurrence or not of significant cooling. In the context of numerical modelling, this means that small inaccuracies in the simulated large-scale freezing level, precipitation intensity, or wind field can already lead to a qualitatively wrong evolution of the temperature field in a valley. Moreover, as just mentioned, operational forecast models for which an eddy-resolving resolution is not feasible will systematically underpredict the volume effect. Thus, deterministic forecasts of cooling by melting in a specific valley will remain very difficult even with substantially improved numerical models. The experience of local forecasters based on the analysis of previous cases is therefore crucial for predicting the snowline in mountainous regions.

Čadež, M.

Fabry, F. and Zawadzki, I.

Findeisen, W.

Jaffé, A.

Lin, C. A. and Stewart, R. E.

Press, W. H., Teukolsky, S. A., Vetterling, W. T. and

Flannery, B. P.

\section{REFERENCES}

1966

1986

1939

1995

1940

1992
Über die Einflüsse des fallenden Schnees auf die Temperatur trockener Atmosphäre. (On the impact of falling snow on the temperature of a dry atmosphere.) Meteorol. Z., 56, 272-273

Long-term radar observations of the melting layer of precipitation and their interpretation. J. Atmos. Sci., 52, 838-851

Die Entstehung der $0^{\circ} \mathrm{C}$-Isothermie und die FraktocumulusBildung unter Nimbostratus. (The formation of a $0^{\circ} \mathrm{C}$ isothermal layer and of fractocumulus clouds beneath a nimbostratus.) Meteorol. Z., 57, 49-54

Über eine Ursache frühsommerlicher Schneefälle in Talniederungen. (On a reason for early-summer snowfall in valleys.) Proc. 9th Internat. Conf. on Alpine Meteorol., Publ. Schweiz. Meteorol. Zentralanstalt, 4, 150-154

Mesoscale circulations initiated by melting snow. J. Geophys. Res., 91, 13299-13302

Numerical Recipes in Fortran77, Vol. 1 of Fortran Numerical Recipes, 2nd edition. Cambridge Univ. Press, UK 
Ralph, F. M., Neiman, P. J. and Rotunno, R.

Steinacker, R.

Steiner, M., Bousquet, O., Houze, R. A. Jr., Smull, B. F. and Mancini, M.

Szeto, K. K., Stewart, R. E. and Lin, C. A.

Szeto, K. K. and Stewart, R. E. Wexler, R., Reed, R. J. and Honig, J.

Zängl, G.
2005 Dropsonde observations in low-level jets over the northeastern Pacific Ocean from CALJET-1998 and PACJET-2001: Mean vertical-profile and atmospheric-river characteristics. Mon. Weather Rev., 133, 899-910

1980 'Über die Ursache sommerlicher Schneefälle in Alpentälern'. (On the reason for summertime snowfall in Alpine valleys.) Pp. 261-265 in Proc. 16th Internat. Conf. on Alpine Meteorol., Aix les Bains, France

1983 Diagnose und Prognose der Schneefallgrenze. (Diagnosing and predicting the snowline.) Wetter und Leben, 35, 81-90

1984 Area-height distribution of a valley and its relation to the valley wind. Contrib. Atmos. Phys., 57, 64-71

2003 Airflow within major Alpine river valleys under heavy rainfall. Q. J. R. Meteorol. Soc., 129, 411-432

1988 Mesoscale circulations forced by melting snow. Part II: Application to meteorological features. J. Atmos. Sci., 45, $1642-1650$

1997 Effects of melting on frontogenesis. J. Atmos. Sci., 54, 689-702

1954 Atmospheric cooling by melting snow. Bull. Amer. Meteorol. Soc., $35,48-51$

2002 An improved method for computing horizontal diffusion in a sigma-coordinate model and its application to simulations over mountainous topography. Mon. Weather Rev., 130, $1423-1432$

2005
Dynamical aspects of wintertime cold-air pools in an Alpine valley system. Mon. Weather Rev., 133, 2721-2740 